\title{
Cardiovascular Risk Assessment among Adults in Primary Health Care Settings in Sulaimani Using WHO Risk Prediction Charts
}

\author{
Vian Najim Edeen \\ Woloba Health Centre \\ Directorate of Health, Sulaimani \\ Ministry of Health \\ Sulaimani, Iraq \\ Email: Najimedeen_vian@yahoo.com
}

\author{
Zhian Salah Ramzi \\ College of Nursing \\ University of Sulaimani \\ Sulaimani, Iraq \\ zean.ramzi@univsul.edu.iq
}

\begin{abstract}
WHO adopted World Health Organization/International Society of Hypertension (WHO/ISH) risk charts to predict cardiovascular disease risk in low and middle income countries. The aim of this study was to estimate the cardiovascular risk in adults $\geq 40$ years old by using the two versions of the WHO/ISH risk prediction charts "with and without cholesterol", and to find out the risk by other parameters not found in the chart; also, to evaluate concordance between the two approaches. A crosssectional study was conducted from August 2016 to February 2017 in two primary health care centers in Sulaimani City among 500 persons aged 40-82 years. The desired information was collected by using a pretested questionnaire; anthropometric measurements and laboratory investigations were also carried out for the participants. Cardiovascular risk was assessed by the WHO/ISH risk prediction charts for the Eastern Mediterranean region in category $D$. Mean age of the participants was $52.5( \pm 9.55)$ years and $70.4 \%$ of the participants were females. Only 260 participants had the results of total cholesterol and triglycerides. The results revealed that, using the risk assessment charts with and without cholesterol, $22.3 \%$ and $19.2 \%$ respectively of the study population were in the tenyear cardiovascular risk category of $\geq 20 \%$. Risk categories were concordant in $86.2 \%$ of the population; and when we applied single risk factor approach 51.5\% would require drug treatment. The WHO/ISH risk chart is an affordable and simple tool to estimate cardiovascular disease risk. Nearly one fifth of adults aged $\geq 40$ years in Sulaimani City are at high-very high risk of developing cardiovascular event in the next ten years as assessed by WHO/ISH risk prediction charts. The use of the without cholesterol version of the chart to estimate cardiovascular disease risk could be useful is settings where cholesterol cannot be measured.
\end{abstract}

Keywords: World Health Organization/International Society of Hypertension (WHO/ISH) risk chart, Cardiovascular risk assessment, Primary health care centers, Sulaimani City.

\section{INTRODUCTION}

Cardiovascular Disease (CVD) is one of the major causes of morbidity and mortality in adults that negatively affects the public health infrastructure, and increases the direct and indirect healthcare costs all over the world [1, 2]. Nearly 17.5 million people died from CVD in 2012 and this number is expected to reach 23.6 million by the year 2030 [3]. About $80 \%$ of deaths from CVD occurred in low and middle income countries (LMIC) usually before the age of 60 [4]. Nearly $10 \%$ of the global disease burden (DALY) is attributed to CVD and this burden is substantially higher in LMIC countries than in high income countries (HIC) [5].

\section{LITERATURE REVIEW}

Cardiovascular Diseases are the diseases of the heart, vascular diseases of the brain and diseases of blood vessels, and deaths from CVD are mainly due to heart attack, stroke and heart failure [6]. Cardiovascular diseases are caused by a range of modifiable and nonmodifiable risk factors; the foremost are age, smoking, hypertension, diabetes and dyslipidemia. These factors are prone to be changed and be controlled by health counseling $[7,8]$. Since multiple factors are responsible for the causation of CVD, so it will be wrong to depend on single risk factor in our assessment, and evidence proposed that CVD risk assessment will be more accurate when we depend on a combination of multiple risk factors than depending on single risk factor $[9,10]$. The total risk approach was first used in developed nations depending on cohort data from their own populations and the most commonly used score is the Framingham Risk Score (FRS), other similar scores and studies are the Munster study, the PROCAM study, the SCORE study, the UKPDS tool, Progetto COURE study and the most recent score is the NHANES [11- 14]. These scores cannot be applied in developing countries since the populations of these countries were not included in the development of any of the abovementioned scores $[15,16]$.

The World Health Organization (WHO) and the International Society of hypertension (ISH) have 
developed CVD risk prediction charts in the year 2000 to be used in different parts of the world. For the development of these charts, WHO and ISH used the best available mortality and risk factor data. It is a simple and cost effective tool to estimate CVD risk for people $\geq 40$ years in primary health care settings in all the 14 WHO sub regions and it shows the risk's percentage for developing CVD event in the next ten years. The simplicity and the cost effectiveness of these charts are due to the fact that they can be used by physician and non-physician health workers $[11,17]$.

According to WHO and World Bank income classification, Iraq is a middle-income country that falls into the Eastern Mediterranean region in category D. The challenge in health sector is the non-communicable diseases (NCD) and mainly CVDs, they are responsible for $26.5 \%$ of all cause of death and are the second cause for disability adjusted life years (DALY) lost in 2012 $[14,17,18]$.

The WHO/ISH risk prediction charts can assist the prevention of CVD, particularly if they can be used by health workers in primary health care settings, because in many LMIC the non-physician health worker is the first contact for medical assistance. Health systems in LMIC do not have the basic infrastructure facilities to use risk scores that are utilized by HIC to predict CVD risk, particularly in primary health care settings. As the WHO/ISH charts use simple variables, they can be applied even in low resource-settings [19].

The present study was carried out to determine the distribution of CVD risk in adults' $\geq 40$ years by using the WHO/ISH risk prediction charts with and without cholesterol in Sulaimani City, and to compare the concordance between the two versions of the chart by using the version of cholesterol as the gold standard; also, to determine the proportion of people who are in need of treatment.

\section{METHODS AND MATERIALS}

From August 2016 to February 2017, a cross-sectional study was conducted in two primary health care centers (PHC) in Sulaimani City, Ibrahim Ahmed and Wooloba PHCs among adults aged 40 years and more. There are 40 PHC in Sulaimani City Center and these PHC were divided into two groups, those who deliver their services to low socioeconomic districts and the second group was those PHCs that are located in districts with middle- high socioeconomic standards of living. One PHC was selected randomly from each of the above 2 categories; Wooloba PHC was chosen randomly to represent the low socioeconomic region and Ibrahim Ahmed PHC was also selected randomly to represent the middle- high socioeconomic region.

Sample size was estimated using sample size equation, to detect the prevalence of $12.6 \%$ of diabetes (CVD risk factor with the lowest prevalence), the minimum sample needed was 481 .

All people who attended the PHC who were $\geq 40$ years males and females had been invited to participate in the study. Persons with established atherosclerotic CVD and pregnant women were excluded from the study. Overall 500 subjects took part in the assessment.
A semi-structured questionnaire was used for the collection of data from each participant after obtaining the verbal consent. Before the usage of these questionnaires, they were piloted on 30 individuals. Height, weight and the waist circumference (anthropometric measurements) were taken for each participant, blood pressure was also measured, blood sugar (fasting or random) was estimated by the researcher using a glucometer. Lipid profile is not one of the routine investigation in all the PHC in Sulaimani, so the results of total cholesterol and triglycerides were obtained by sending all the participants to Sulaimani Public Health Laboratory. Cardiovascular risk was estimated for each participant by using the WHO/ISH risk prediction chart for the Eastern Mediterranean Region in category D. the variables that we depend on the prediction of CVD risk were age, gender, systolic blood pressure, smoking status, the presence or absence of diabetes; these variables were used for the version of the chart without cholesterol and with the addition of serum cholesterol level for the version of the chart with cholesterol.

Blood glucose has been measured by a glucometer and study participants were classified as diabetics based on the definition of WHO and the diagnostic criteria of the American Diabetic Association (ADA) for fasting blood sugar of $7 \mathrm{mmol} / \mathrm{dl}(>126 \mathrm{mg} / \mathrm{dL})$ and/or random blood sugar $11.1 \mathrm{mmol} / \mathrm{l}(>200 \mathrm{mg} / \mathrm{dL})$ [20, 21]. In addition, participants that were previously diagnosed as having diabetes and who were on treatment were also classified as diabetic regardless their blood glucose status. Blood pressure was measured for the participants and they were labeled as hypertensive when systolic blood pressure $\geq 140 \mathrm{~mm} \mathrm{Hg}$ and/or diastolic blood pressure $\geq 90 \mathrm{~mm}$ $\mathrm{Hg}$ or in case they were on antihypertensive medication (14). Waist circumference (WC) was measured in line halfway between the lowest rib and the iliac crest using a regular tailor tape. According to the measurement, respondents were grouped into 3 categories: normal, elevated, and substantially elevated WC (SEWC). This categorization followed sex-specific cut-off points suggested by WHO: elevated WC indexed as a waistline measurement of $94 \mathrm{~cm}$ or more for men and $80 \mathrm{~cm}$ or more for women, and SEWC indexed as a waistline measurement of $102 \mathrm{~cm}$ or more for men and $88 \mathrm{~cm}$ or more for women [22].

IBM SPSS Statistics 21 was used for data entry and analysis. Chi-square test was used for statistical significance with a threshold of $\mathrm{p} \leq 0.05$. The method described in the WHO guideline was used for data analysis [1]. Concordance between the two prediction charts was calculated for the different risk levels using the chart with cholesterol as gold standard.

\section{RESULTS}

A total of 500 participants (aged 40-82 years), mean age was $52.5( \pm 9.55)$ years, $70.4 \%$ of the participants were females; nearly half (47\%) of the participants were from the age group 40-49 years (Table 1). 
Table 1: Age and gender distribution of the study participants

\begin{tabular}{|l|c|c|c|l|l|l|}
\hline \multirow{3}{*}{ Gender } & \multicolumn{7}{|c|}{ Age group (years) } \\
\cline { 2 - 7 } & $40-49$ & $50-59$ & $60-69$ & $\geq 70$ & $\begin{array}{l}\text { Total } \\
\text { n (\%) }\end{array}$ & Mean \pm SD \\
\hline \multirow{3}{*}{ Male } & 62 & 40 & 33 & 13 & 148 & $53.53 \pm 9.57$ \\
\hline & $(26.4)$ & $(31)$ & $(32.4)$ & $(38.2)$ & $(29.6)$ & \\
Female & 173 & 89 & 69 & 21 & 352 & $52.07 \pm 9.52$ \\
$(73.6)$ & $(69)$ & $(67.6)$ & $(61.8)$ & $(70.4)$ & \\
\hline $\begin{array}{l}\text { Total } \\
(\%)\end{array}$ & 235 & 129 & 102 & 34 & 500 & $52.50 \pm 9.55$ \\
$(47)$ & $(25.8)$ & $(20.4)$ & $(6.8)$ & $(100)$ & \\
\hline
\end{tabular}

About two thirds of the participants were from the low income PHC (Wooloba PHC), nearly 83\% of the participants were married and about $52 \%$ were illiterate. Housewives represented around $63 \%$ of the study participants. Mean number of family members was 5.34( \pm 2.35$)$ and mean crowding index was $1.26( \pm 0.63)$.

Table 2 shows the prevalence of CVD risk factors that are used in the assessment of CVD, in which fasting blood cholesterol $\geq 5.18 \mathrm{mmol} / \mathrm{L}$ showed the highest prevalence of $45.8 \%$, followed by systolic blood pressure $\geq 140 \mathrm{mmHg}(41.8 \%)$, then diabetes showed a prevalence of $17.4 \%$ and at last smoking with a prevalence of $8.8 \%$. In addition to the above-mentioned CVD risk factors, age and gender should also be noted as they are two important demographic characteristics used in WHO/ISH risk prediction charts.

Mean cigarette index was calculated by using both variables, the number of cigarettes smoked/day variable and the duration of smoking in year variable; it was equal to $27.66( \pm 27.08)$ pack/year.

Diabetic participants represented $12.8 \%$ of the study subjects that was equal to 63 participants. Out of the 437 participants that didn't have diabetes 28 participants (5.6\%) were with undiagnosed D.M (Fig.1).

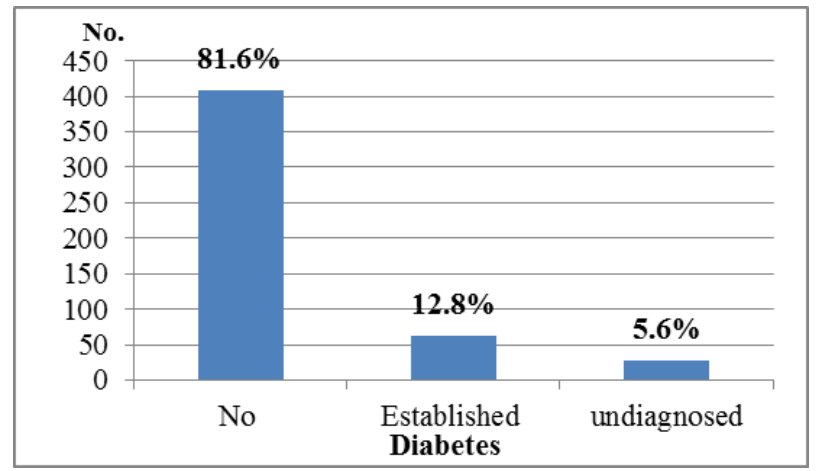

Fig.1 Frequency of diabetes among the participants

The number of participants with established hypertension was 135 participants (27\%); Out of the 365 participants who mentioned that they do not have hypertension, 161 participants (32.2\%) were with undiagnosed hypertension (Fig.2).

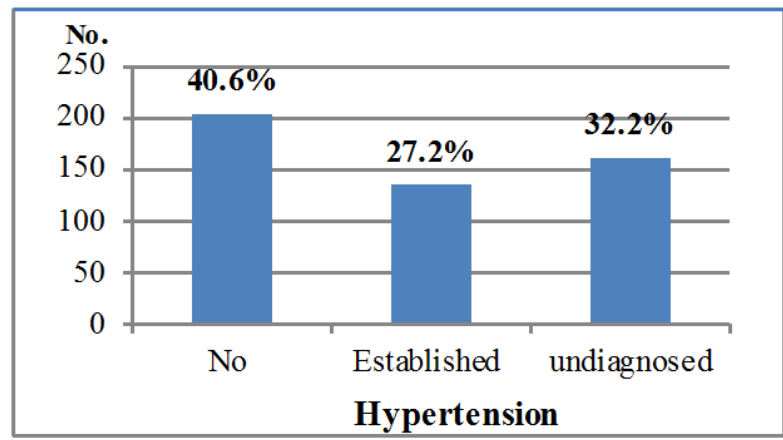

Fig.2 Frequency of hypertension among the participants.

Table 2 reveals the distribution of total CVD risk in the study population by using both sets of the WHO/ISH EMR D risk prediction charts, with and without cholesterol. For risk assessment using the with cholesterol version of the chart, $77.7 \%$ had a ten-year risk $<20 \%$; the equivalent proportion was $80.8 \%$. The proportion of risk $\geq 20 \%$ (high- very high) were $22.3 \%$ and $19.2 \%$ with and without cholesterol respectively. When we used the charts without cholesterol, the percentage of the participants in the high (20-29\% 10 year risk) and very high risk groups $(\geq 30 \%)$ was less than chart with cholesterol (13\% vs $14.6 \%$ and $6.2 \%$ vs $7.7 \%$ respectively). Generally, male had higher CVD risk than females when assessed by both versions of the chart.

Table 2: Ten-year total CVD risk prediction by gender, using WHO/ISH EMR D charts, with and without

\begin{tabular}{|c|c|c|c|c|}
\hline $\begin{array}{l}\text { Sex } \\
\text { No. (\%) }\end{array}$ & ${ }^{\mathrm{a}} \mathrm{HT}$ & ${ }^{\mathrm{b}} \mathrm{DM}$ & Smoking & ${ }^{\mathrm{c}} \mathrm{HC}$ \\
\hline $\begin{array}{l}\text { Male } \\
148 \\
(29.6 \%)\end{array}$ & $\begin{array}{l}76 \\
(51.4)\end{array}$ & $\begin{array}{l}30 \\
(20.3)\end{array}$ & 27 (18.2) & $\begin{array}{l}34 / \mathrm{d} 260 \\
(44.2)\end{array}$ \\
\hline $\begin{array}{l}\text { Female } \\
352 \\
(70.4 \%)\end{array}$ & $\begin{array}{l}133 \\
(37.8)\end{array}$ & $\begin{array}{l}57 \\
(16.2)\end{array}$ & $17(4.8)$ & $\begin{array}{l}85 / 260 \\
(46.4)\end{array}$ \\
\hline $\begin{array}{l}\text { Total } \\
500 \\
(100 \%)\end{array}$ & $\begin{array}{l}209 \\
(41.8)\end{array}$ & $\begin{array}{l}87 \\
(17.4)\end{array}$ & $44(8.8)$ & $\begin{array}{l}\text { 119/260 } \\
(45.8)\end{array}$ \\
\hline
\end{tabular}

In this study, the chart with cholesterol was found to be concordant for 224/260 (86.2\%) with the without cholesterol version of the chart. Without information about cholesterol, there was overestimation of CVD risk in 24 (9\%) and underestimation in12 (4.6\%). Out of the 36 individuals with non-concordant CVD risk estimates, $66.7 \%$ (24/36) were overestimates; 33.3\% (12/36) related to low and moderate CVD risk groups that would not have drug intervention at a CVD risk threshold risk $\geq 20 \%$ with either chart. Only $2.7 \%$ (7/260) of the total sample was misclassified regarding the need for drug intervention: risk was overestimated in 1.9\% (5/260) and underestimated in $0.8 \%$ (2/260) (Table 3). 
Table 3: CVD risk factors prevalence in association with gender

\begin{tabular}{|c|lc|ll|l|l|l|}
\hline 10-year WHO/ISH & \multicolumn{5}{|c|}{ Gender } & & \\
\cline { 2 - 8 } cardior ascular risk prediction & Male & & Female & Total \% & P-rahue \\
\hline With cholesterol & & & & & & \\
$<10 \%$ (Low) & 50 & 64.9 & 139 & 76 & 189 & 72.7 & \multirow{2}{*}{0.279} \\
$10-<20 \%$ (Moderate) & 6 & 7.8 & 7 & 3.8 & 13 & 5 & \\
\hline $20-<30 \%$ (High) & 14 & 18.2 & 24 & 13.1 & 38 & 14.6 & \\
\hline$\geq 30 \%$ (Very high) & 7 & 9.1 & 13 & 7.1 & 20 & 7.7 & \\
Total & 77 & 100 & 183 & 100 & 260 & 100 & \\
Without cholesterol & & & & & & & \\
$<10 \%$ (Low) & 85 & 57.4 & 282 & 80.1 & 367 & 73.4 & $<0.001$ \\
\hline $10-<20 \%$ (Moderate) & 20 & 13.5 & 17 & 4.8 & 37 & 7.4 & \\
\hline $20-<30 \%$ (High) & 20 & 13.5 & 45 & 12.8 & 65 & 13 & \\
$\geq 30 \%$ (Very high) & 23 & 15.5 & 8 & 2.3 & 31 & 6.2 & \\
Total & 148 & 100 & 352 & 70.4 & 500 & 100 & \\
& & & & & &
\end{tabular}

Table 4 shows the comparison of drug requirements for CVD prevention between total CVD risk assessment, with and without cholesterol on one hand and single risk factor approach on the other hand. By using WHO guidelines for CVD risk assessment and management (12), the assessed proportions of participants who required medication to reduce CVD risk in our studied sample were different when we used the with and without cholesterol chart. Using a threshold of $\geq 30 \%$, $4.6 \%$ of the participants studied without measuring cholesterol were regarded as in need for drugs; while when we used a threshold of $\geq 20 \%$ risk of developing CVD, 20.4\% of the studied participants required drugs. For the chart with cholesterol the respective proportions were $7.7 \%$ and $22.3 \%$. When we applied the single risk factor approach, 158 out of the total 260 participants (51.5\%) would require drug treatment (22.3\% antihypertensive drugs and 29.2\% lipid-lowering drugs); almost tenfold higher than without cholesterol and sixfold higher than with cholesterol risk approach.

Table 4: Concordance of WHO/ISH EMR D CVD risk charts with and without cholesterol.

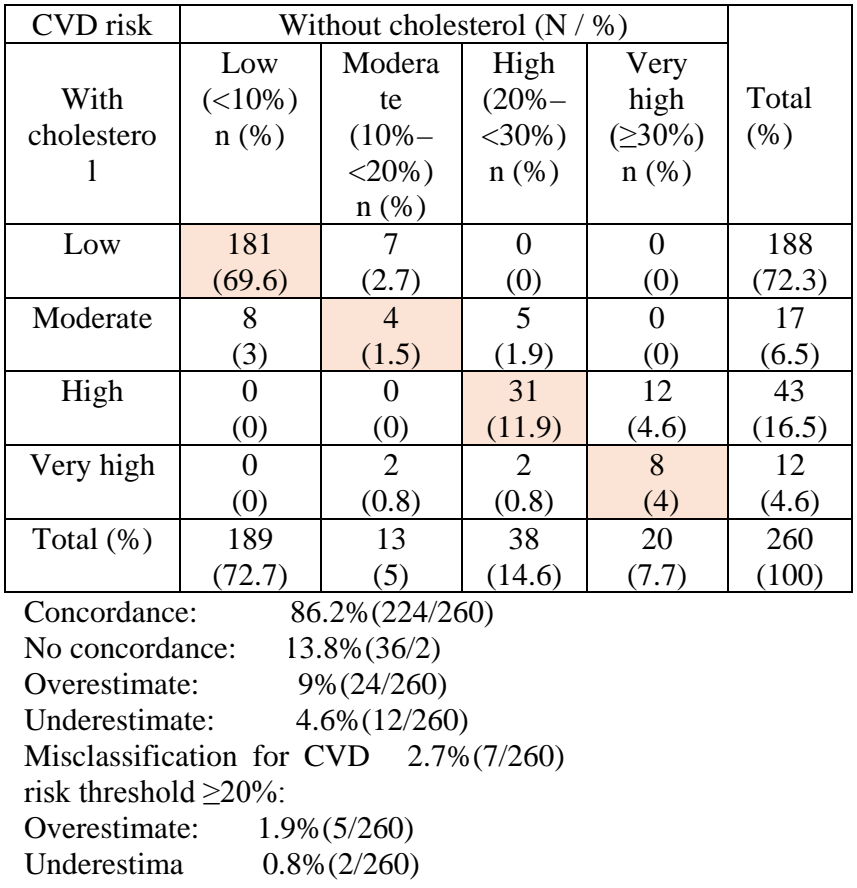

\section{DISCUSSION}

This study is the first in Iraq and especially in Kurdistan that used the WHO/ISH risk charts "with and without" cholesterol for the prediction of CVD event. It revealed that about $20 \%$ of adults aged $\geq 40$ years in Sulaimani City at high- very high risk of developing CVD in the next ten years, and one third of these individuals are in the very high risk category. These proportions are nearly the same in case the "with or without" cholesterol version of the chart is used. The proportions in this study seem to be very high if we compare it with that observed by Mendis in eight LMICs (1.1\%-10\%) [23], by Khanal in Nepal (4.3\%) (24), by Fatema in Bangladesh (4.6\%) [5], by Otogontuya in Mongolia (6\%) [25], and by Ghorpade in India (10\%) [26]. We found significant differences between estimates without cholesterol version of the chart, but estimates with cholesterol version of the chart showed no significant difference. The main aim of these charts is to identify people at high risk who need immediate intervention, and this aim was achieved in our study by the version of the chart without cholesterol.

Our study revealed that $5.6 \%$ of the study participants had undiagnosed diabetes. Basically because of the epidemiological transition that has been experienced in this country; also, due to the fact that screening for diabetes in developing countries is difficult because of limited resources, blood tests may be challenging, and we may need to depend on inexpensive tests available to predict persons at high risk of developing diabetes [27, 28]. Significant number of participants (32\%) with hypertension were unaware of their condition; it is higher than the rates of a recent study that was done in Cairo (Egypt) (11\%) [29], but it is less than of that done in India (40\%) [30].

Finding out the level of agreement between the results achieved by the "with and without" cholesterol versions of the WHO/ISH risk prediction chart was the heart of this study. The percentage of concordance in this study (86.2\%) was similar to that of a Cuban study [31] in which the percentage of concordance was $88.1 \%, 10.6 \%$ overestimation and $1.3 \%$ underestimation. It is also similar to that of a study that was carried out in Bangladesh [15]; they found 89.5\% concordance, 7.6\% overestimation and $1.1 \%$ underestimation among the non-concordant. But it was higher than that reported by Green in Washington (USA) applying Framingham CVD risk scoring using cholesterol and/or BMI formulae. They found $78.2 \%$ concordance, $20.3 \%$ overestimation and $1.4 \%$ underestimation among the no concordant [32,33,34,35].

This study showed that using the version of the chart without cholesterol would result in only $1.9 \%$ of the participants receiving drugs due to overestimation, a figure lower than that of the two studies that were done in Cuba and Bangladesh (2.2\% and 3.4\% respectively). The results of this study also revealed that $0.8 \%$ not receiving them due to underestimation, which indicated that when we use the CVD version without cholesterol, the risk of error in leaving a person in need for intervention without pharmaco-prevention is quite small. The Cuban study showed lower figures than that of our study (0.5\%); but the study that was done in Bangladesh 
showed higher figures than ours in which $1.1 \%$ didn't receive drugs due to underestimation.

The agreement between the two versions of the chart was further investigated by a practical technique depending on the need for medication as indicated by the two versions of the WHO/ISH risk chart. It was clear that, compared to single risk factor approach, a considerably less proportion of participants would require medication if they were assessed by the WHO/ISH risk chart. Nevertheless, the percentage of the participants was about sixfold to tenfold higher (when the threshold of the absolute risk is $\geq 30 \%$ ), and the percentage of participants was about 2-2.5 times higher (when the threshold of the absolute risk is $\geq 20 \%$ ) when suggestion is made by the with cholesterol version in comparison with the without cholesterol version of the chart. In Bangladesh [15], the percentage of participants was about fivefold to sevenfold higher (when the threshold of the absolute risk is $\geq 30 \%$ or $\geq 20 \%$ ), but in Cuba [31] the single risk factor approach would more than triple drug usage if we compare it to the need for drug treatment as assessed by the two versions of the WHO/ISH charts. These differences of the findings from that of our study may be attributed to the small sample size (260 participants) as compared to Bangladesh (sample size=563) and Cuba (sample size=1287).

Reducing the threshold of CVD risk from $\geq 30 \%$ to $\geq 20 \%$ didn't obviously increase drug requirement, but it increased the ability to detect persons at risk, as established by the Mendis eight LMICs study [23]; the same results was also found by the Cuban study [31, 32] and the study that was done in Bangladesh [14]. However, the assessment of CVD using both versions of the chart better targeting persons who are prone to develop CVD, so the number of participants in need of drugs will be limited; in contrast to single risk factor approach that showed overestimation i.e., more need for drugs in large proportion of participants.

This study revealed that using the without cholesterol version of the chart enabled us to diagnose participants who are at high CVD risk, hence we will be able to take the right steps in managing them; this is substantially important because the majority of primary health setting in LMICs lack testing for cholesterol. Since this study is the first to use the WHO/ISH in primary health care setting in Sulaimani City; similar studies in other PHC in Kurdistan and especially in Sulaimani City will be needed to confirm the findings of our study.

In addition, there is no cohort or any other longitudinal studies of CVD risk factors regarding morbidity and mortality in almost all LMICs, therefore no justified or calibrated cardiovascular risk scores have been developed for their populations.

\section{CONCLUSION}

In conclusion, about one fifth of adults aged $\geq 40$ years in Sulaimani City at high- very high risk for developing CVD in the next 10 years, and one third of these individuals are at very high risk, with males being more vulnerable to develop CVD event than females. The very high concordance between the two versions of the chart (with and without cholesterol versions) indicates that the use of the without cholesterol version of the chart is an effective tool to focus on persons who are more prone to develop CVD, without causing overuse of drugs for the prevention of CVD event; so even if we have limited resources and poor laboratory facilities, the without cholesterol version of the chart is an effective tool to be used in primary health care setting in LMICs for better targeting of persons who are at high risk for developing CVD event. The results of the study also signify that a wide scale study will be needed to corroborate these findings.

\section{REFERENCE}

[1] World Health Organization. "Prevention of cardiovascular disease: Guidelines for assessment and management of cardiovascular risk," Geneva, WHO, 2007.

[2] DO. Abegunde, CD. Mathers, T. Adam, M. Ortegon, K. Strong, "The burden and costs of chronic diseases in low-income and middle-income countries,” Lancet; 370 (9603), pp. 1929-38, 2007.

[3] American Heart Association, "Heart Disease and Stroke Statistics - At-a-Glance” onebraveidea.com, Jan. 25, 2017 [Online]. Available:

http://www.onebraveidea.com/submissions/ucm_470704.pdf. [Accessed: 2017].

[4] World Health Organization, "World Heart Federation, World Stroke Organization,” Global Atlas on cardiovascular disease prevention and control, 2011.

[5] World Health Organization, “The global burden of disease 2004 update, 2008.

[6] Heart Information Center, "Heart Anatomy, www.texasheart.org August $2016 \quad$ [Online] Available:http://www.texasheart.org/HIC/Anatomy/anatomy2. cfm. [Accessed: 2016].

[7] BA. Raphael, AA. Ernest, A. Charles, "Chronic NonCommunicable Diseases in Low and Middle-income,” 2016.

[8] World Health Organization, "Global Health Risks: Mortality and Burden of Disease Attributable to Selected Major Risks," 2009.

[9] R. Jackson, CM. Lawes, DA. Bennett, RJ. Minle, A. Rodgers, "Treatment with drugs to lower blood pressure and blood cholesterol based on an individual's absolute cardiovascular risk,” Lancet, 365(9457), pp. 434-41, 2005.

[10] WB. Kannel, D. McGee, "Diabetes and glucose tolerance as risk factors for cardiovascular disease: the Framingham study,” Diabetes Care, 2(2), pp. 120-6, 1979.

[11] S. Mendis, L.H. Lindholm, G. Mancia, J. Whitworth, M. Alderman, S. Lim, et al., "World Health Organization (WHO) and International Society of Hypertension (ISH) risk prediction charts: assessment of cardiovascular risk for prevention and control of cardiovascular disease in low and middle-income countries,” J Hypertens, 25 (8), pp. 15781582, 2007.

[12] RB. Agostino, RS. Vasan, MJ. Pencina, PA. Wolf, M. Cobain, JM. Massaro, et al., "General cardiovascular risk profile for use in primary care: the Framingham Heart Study," Circulation, 117(6), pp. 743-53, 2008.

[13] XF. Zhang, J. Attia, C. D’Este, XH. Yu, XG. Wu, “A risk score predicted coronary heart disease and stroke in a Chinese cohort,” J Clin Epidemiol, 58(9), pp. 951-82005.

[14] World Health Organization, "Prevention of Cardiovascular Disease: Pocket guidelines with cardiovascular risk prediction charts for assessment and management of cardiovascular risk," 2007.

[15] K. Fatema, NA. Zwar, AH. Milton, B. Rahman, L. Ali, "Application of two versions of the WHO/international society of hypertension absolute cardiovascular risk assessment tools in a rural Bangladeshi population,” BMJ Open, 5(10), pp. 10, 2015.

[16] JA. Batsis, F. Lopes- Jimenez, “Cardiovascular risk assessment From individual risk prediction to estimation of global risk and change in risk in the population," BMC Medicine, 29 (8), pp.12, 2010.

[17] World Health Organization, "WHO/ISH Risk prediction charts for 14 WHO epidemiological sub-regions, ish-world.com, Nov. 19, 2014. [Online]. Available: http://ish- 
world.com/downloads/activities/colour_charts_24_

Aug_07.pdf. [Accessed: 2007].

[18] World Health Organization, "Iraq: WHO statistical profile," who.int, Jan 2015. [Online]. Available: http://www.who.int/gho/countries/irq.pdf. [Accessed: 2012].

[19] World Health Organization, "International Society of Hypertension. Cardiovascular risk prediction charts: strengths and limitations,” who.int, Feb. 2012. [Online]. Available: http://www.who.int/cardiovascular_diseases/publications/cvd_ qa.pdf?ua=1. [Accessed: 2013.

[20] World Health Organization, "Definition and Diagnosis of Diabetes Mellitus and Intermediate Hyperglycemia,” 2006.

[21] Mayo Clinic, “Type 2 diabetes Diagnosis,” mayoclinic.org, Aug. 23. 2017 [Online]. Available: http://www.mayoclinic.org/diseases-conditions/type-2diabetes/diagnosis-treatment /diagnosis/dxc-20169894. [Accessed: 2017].

[22] World Health Organization, "Waist circumference and waist-hip ratio,” 2008.

[23] S. Mendis, LH. Lindholm, SG. Anderson, A. Alwan, R. Koju, BJ, Onwubere. et al., "Total cardiovascular risk approach to improve efficiency of cardiovascular prevention in resource constrain settings,” J Clin Epidemiol, 64(12), pp. 1451-62, 2011.

[24] MK. Khanal, M. Ahmed, M. Moniruzzaman, PC. Banik, RR. Dhungana, P. Bhandari, et al., "Total cardiovascular risk for next 10 years among rural population of Nepal using WHO/ISH risk prediction chart,” BMC Res Notes, 10(1), pp. 120-8, 2017.

[25] S. Otgontuya, BS. Oum, R. Buckley, “Assessment of total cardiovascular risk using WHO/ISH risk prediction charts in three low and middle income countries in Asia," BMC Public Health, 539 (13), pp. 312-24, 2013.

[26] GA. Ghorpade, SR. Shrivastava, SS. Kar, S. Sarkar, SM. Majidi, G. Roy, "Estimation of the cardiovascular risk using World Health Organization/International Society of Hypertension (WHO/ISH) risk prediction charts in a rural population of South India,” Int J Health Policy Manag, 4(8), pp. 531-36, 2015.

[27] Stockholm International Peace Research Institute. World Diabetes Day - Diabetes in conflict areas sipri.org, Jul. 2012. [Online]. Available: https://www.sipri.org/commentary/blog/2012/world-diabetesday-diabetes-conflict-areas. [Accessed: 2012].

[28] A. Mansour, F. Al Douri, "Diabetes in Iraq: Facing the Epidemic. A systematic Review,” Wulfenia Journal, 22(3), pp. 258-73, 2015.

[29] M. Abd Elaziz, SA. Dewedar, S. Sabbour, MM. El Gafaary, DM. Marzouk, A. Aboul Fotouh, et al., "Screening for hypertension among adults: community outreach in Cairo,” Egypt. J Public Health (Oxf), 37(4), pp. 701-6, 2014.

[30] AN. Shukla, T. Madan, BM. Thakkar, MM. Parmar, KH. Shah, "Prevalence and Predictors of Undiagnosed Hypertension in an Apparently Healthy Western Indian Population,” $A d v$ Epidemiol.(Hindawi), 5(4), pp. 6491-8, 3015.

[31] P. Nordet, S. Mendis, A. Dueñas, R. de la Noval, N. Armas, IL. de la Noval, et al., "Total cardiovascular risk assessment and management using two prediction tools, with and without blood cholesterol,” MEDICC Rev, 15(4), pp. 36-40, 2013.

[32] BB. Green, ML. Anderson, AJ. Cook, S. Catz, PA. Fishman, JB. McClure, et al., "Using body mass index data in the electronic health record to calculate cardiovascular risk," Am J Prev Med, 42(4), pp. 342-7, 2012. 